\title{
Single nanoparticle as photonic switch and optical memory element
}

\author{
B. F. Soares, K. F. MacDonald, V. A. Fedotov, M. Bashevoy, and N. I. Zheludev \\ EPSRC NanoPhotonics Portfolio Centre, School of Physics and Astronomy, University of Southampton, SO17 1BJ, UK \\ Tel. +44(0)23 80593566, fax. +44(0)23 80593910, e-mail n.i.zheludev@soton.ac.uk
}

\begin{abstract}
We suggest and experimentally demonstrate a new concept for optical nanowatt switching and memory function made possible by light-induced structural transformations in a single nanoparticle.

(C)2005 Optical Society of America

OCIS codes: (190.3970) Microparticle nonlinear optics, (070.4340) Nonlinear optical signal processing
\end{abstract}

In this paper we analyze the potential for using a single nanoparticle undergoing a structural transformation as the key element of low energy consumption nanophotonic devices. In essence, this new opportunity relies on the fact that in contrast with bulk solids where phase transitions are abrupt and characterized by the precise coexistence of different structural phases, structural transformations in nanoparticles proceed through a dynamic coexistence of structural forms - making the transition continuous and reversible. When a nanoparticle is brought to the verge of what would be a first-order structural phase transition in the bulk, its structural composition can become significantly more sensitive to external stimulation. If the structural phases involved have different optical properties, the continuous structural transformation will be accompanied by a change in the dielectric properties of the nanoparticle, thus providing an optical nonlinearity $[1,2]$. This is illustrated in Fig. 1 using the example of a nanoparticle undergoing a surface-driven phase transition from a low-temperature phase (white) to a highertemperature phase (grey). This transition may be activated by increasing the ambient temperature, or by external optical or electron-beam excitation at fixed ambient temperature. In the later case, increasing the level of excitation will sequentially convert the particle from state $I$ to state $I V$. However, if the excitation is withdrawn at any point before state $I V$ is achieved, the particle will return to state $I$. If the structural phases involved in the transition have substantially different properties, the particle's overall optical and electronic properties will vary as a function of excitation intensity as shown schematically by the graph in Fig. 1.
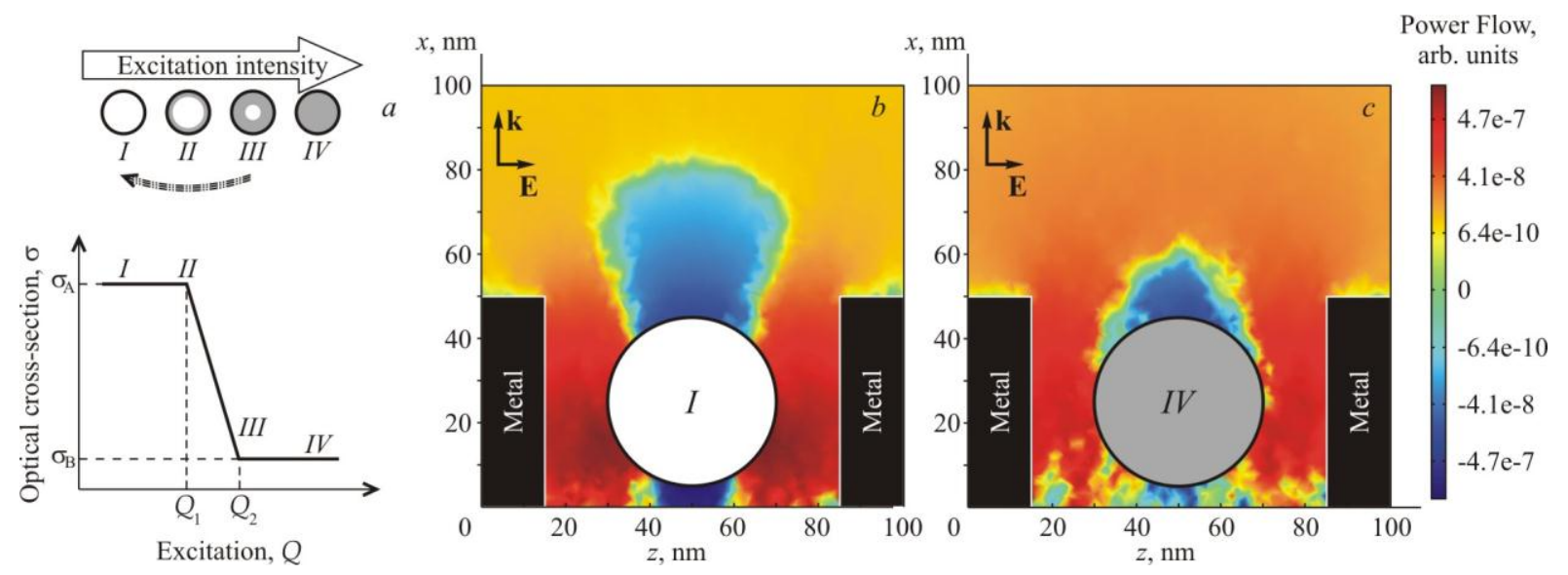

Fig. 1. (a) Continuous coexistence of structural phases in a nanoparticle undergoing a light-induced structural transformation and corresponding dependence of the particle's optical cross-section on excitation intensity. (b and c) 3D finite-element calculations of the power flow distribution for a $70 \mathrm{~nm}$ metal aperture containing a $40 \mathrm{~nm}$ gallium nanoparticle in (b) a low-temperature, high optical cross-section phase, and (c) a high-temperature, low optical crosssection phase (400 $\mathrm{nm}$ light incident from below).

A nanoparticle undergoing a structural transformation could offer a variety of applications in nanophotonic devices. These functionalities may be achieved if the particle is placed in an optical nano-aperture, used as an element of a chain waveguide, or employed as a scattering center in a photonic-bandgap or plasmon-polariton 
waveguide. Here we will consider the generic case of a nanoparticle placed in a nano-aperture, as illustrated in Fig. 2. Stimulating the particle with external excitation will change its optical cross-section thus modulating the aperture's transmission and reflection, creating an optical switch (see Fig.'s $2 \mathrm{a}$ and b).

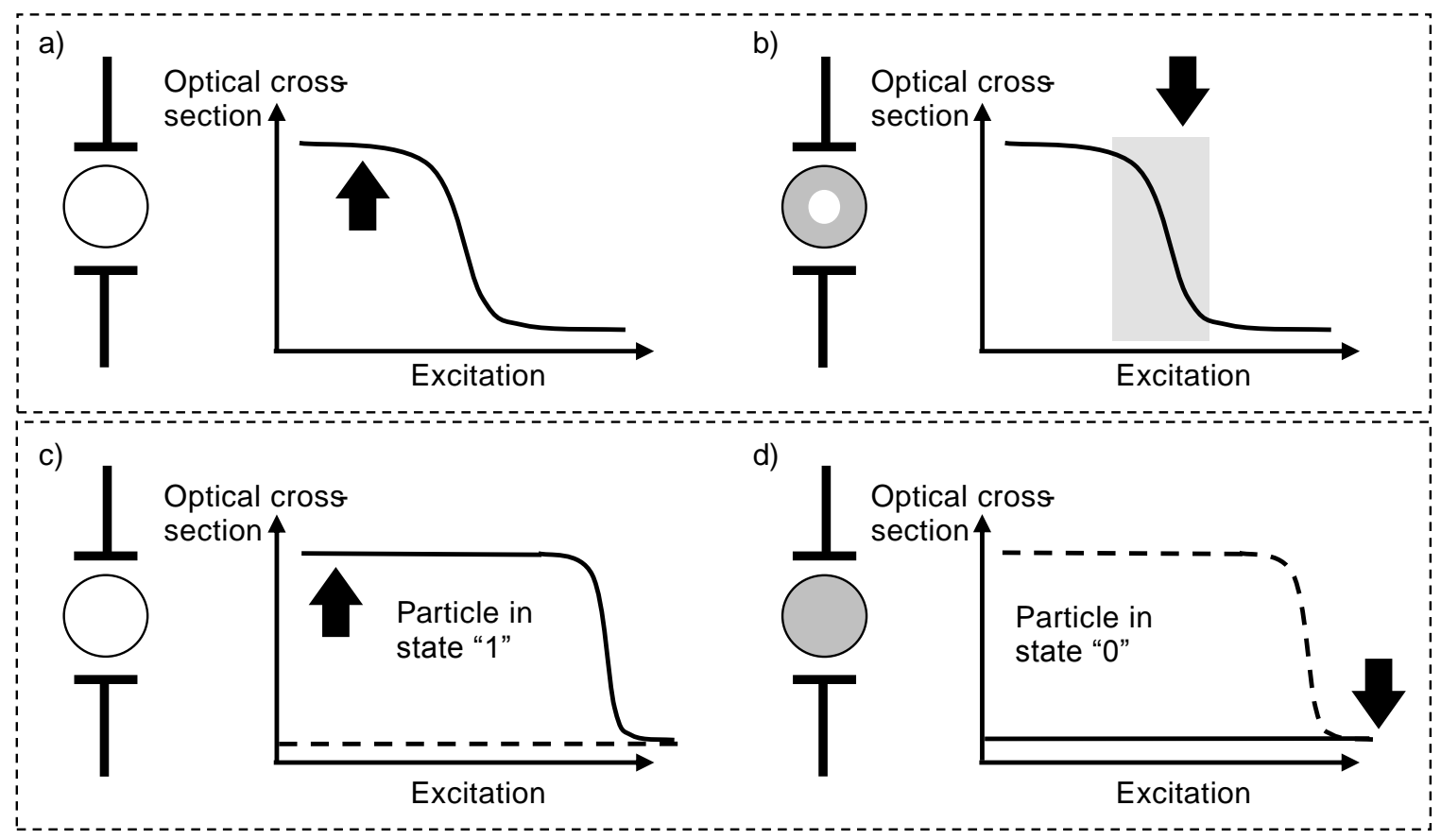

Fig. 2. Nanoparticle undergoing a structural transformation in a nano-aperture. (a) and (b) Optical switch function; (c) and (d) optical memory function

A remarkable characteristic of a nanoparticle undergoing a phase transition is that it shows a structural hysteresis: when the ambient temperature or excitation intensity exceeds the level required for complete transformation of the particle into the high-temperature phase, its return to the low-temperature phase requires overcooling. Thus a nanoparticle is inheritably a bistable object and presents the possibility of creating an optical memory element (see Fig.'s $2 \mathrm{c}$ and d). In the low-temperature phase with optical cross section $\sigma_{A}$ the nanoparticle is in memory state " $O$ ". When sufficiently strong optical excitation is provided the particle goes into the hightemperature state " 1 " with optical cross section $\sigma_{B}$ and remains there until the ambient temperature is reduced below the overcooling limit. However, if the particle is not switched into state " 1 " by optical excitation it will remain in state " $O$ " as long as the ambient temperature remains below the transition temperature. The state of the nanoparticle may be read using a weak probe beam, thus enabling it to act a basic optical memory element.

Here we illustrated the above outlined concept with the first experimental demonstration of this phasetransition switching concept on a single nanoparticle. We have observed a sequence of light-induced structural phase transformations involving five different phases in a single gallium nanoparticle. We found that these transformations are achieved through the controllable, continuous and reversible coexistence of different crystalline and disordered forms and can be stimulated by extremely low power optical excitation. We have been able to induce and monitor transitions between phases that differ in free energy by only a fraction of an $\mathrm{meV}$ per atom and found that the nanoparticle's structural response to optical excitation settles within a few tens of microseconds. We also observed that a particle probed with low intensity $\mathrm{cw}$ laser light can be overcooled by more than $90 \mathrm{~K}$ before it returns to the low-temperature phase, thus demonstrating the hysteresis characteristic necessary for the optical memory element.

We studied a single nanoparticle grown from an atomic beam at the tip of a tapered optical fiber with a nanoaperture at its end. This location allows for precise coupling of optical excitations to the particle for the stimulation of phase transformations, and simultaneously for collection of a probe signal reflected by the particle and used to monitor its state. Structural transformations were observed by monitoring the reflectivity of the particle and pumpbeam-induced changes in reflectivity as functions of temperature between 80 and $300 \mathrm{~K}$ (varied at a rate of $\sim 2$ 
$\mathrm{K} / \mathrm{min}$ ) using pump and probe powers of 30 and $20 \mathrm{nW}$ respectively at the nano-aperture. While increasing the temperature of the nanoparticle we observe several narrow peaks in the pump-probe signal, as shown in Fig. 3. Positive and negative peaks show pump-induced increases and decreases in nanoparticle reflectivity corresponding to the following sequence of structural transformations in the gallium particle: $\gamma \rightarrow \varepsilon \rightarrow \delta \rightarrow \beta \rightarrow$ liquid (Greek letters denote different structural phases according to the standard classification of gallium's crystalline forms).

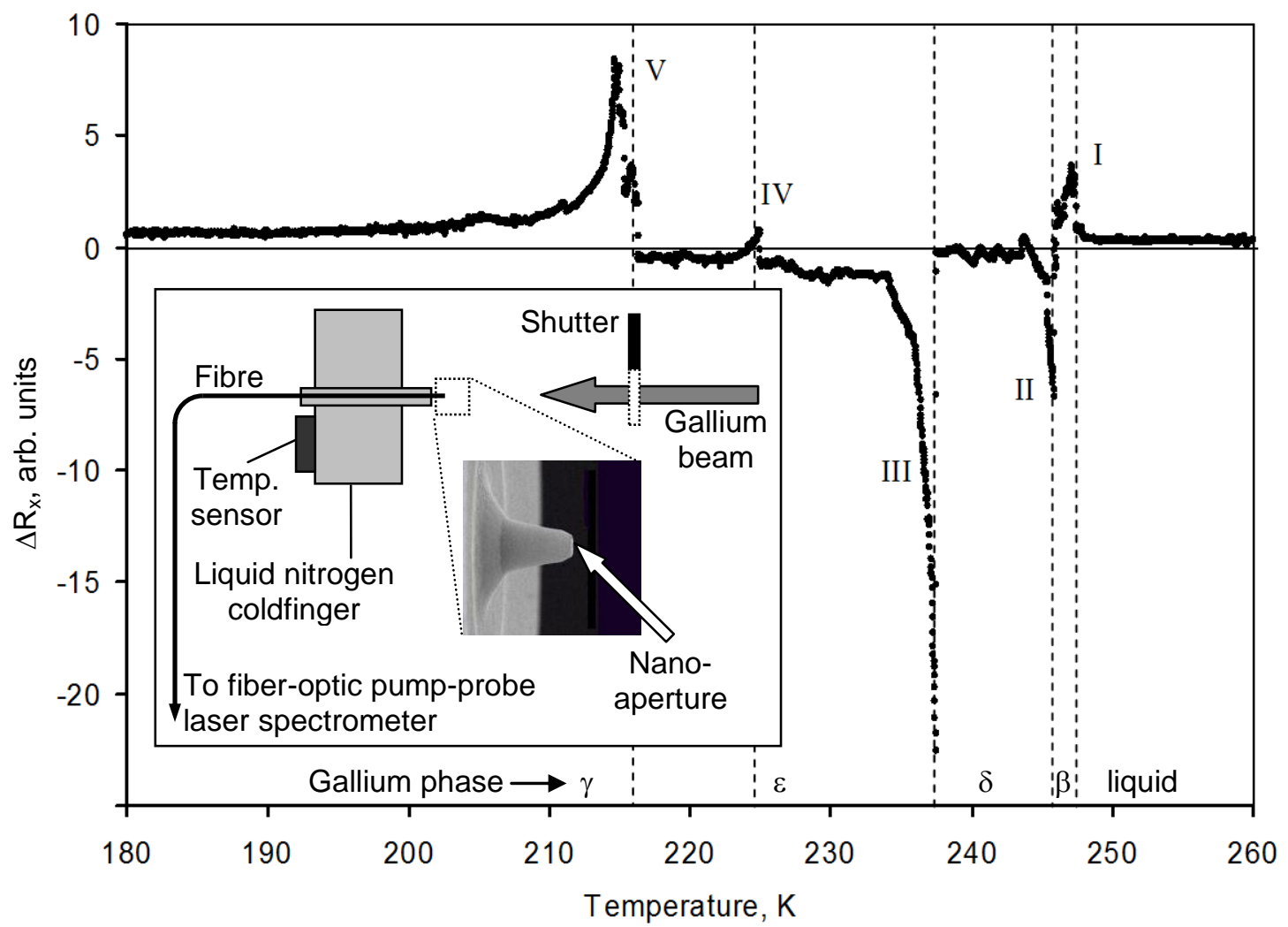

Fig. 3. Light-induced structural transformations in a single gallium nanoparticle are indicated by peaks in the magnitude $\left(\Delta R_{x}\right)$ of pump-induced reflectivity change as a function of increasing temperature (cw probe power at particle $\sim 20 \mathrm{nW}$, pump peak power $\sim 30 \mathrm{nW}$ ). Inset is a schematic of the experimental configuration of the tapered fiber tip where the nanoparticle is formed by atomic beam deposition under high vacuum.

The light-induced structural transitions are observed at very low levels of optical excitation. Such levels can be used because the differences $\Delta G$ between the free energies of some of the metastable phases involved are very small: for example, $\Delta G_{\delta \cdot \beta}=0.3 \mathrm{meV} /$ atom and $\Delta G_{\gamma \delta}=17 \mathrm{meV} /$ atom. Thus, the absorption of a 'pump' quantum with an energy of $0.8 \mathrm{eV}$ should be sufficient to convert about 500 atoms from the $\gamma$ phase to the $\delta$ phase, and about 2700 atoms from the $\delta$ phase to the $\beta$ phase. The light-induced transitions are likely to be driven primarily by thermal excitation (i.e. laser-induced heating) but there may also be a contribution from a temperature-independent, non-thermal mechanism where the phase change is caused by band-structure collapse and lattice instability resulting from strong electronic excitation.

[1] K. F. MacDonald, V. A. Fedotov, S. Pochon, G. C. Stevens, F. V. Kusmartsev, V. I. Emel'yanov, and N. I. Zheludev, "Controlling the coexistence of structural phases and the optical properties of gallium nanoparticles with optical excitation." Europhys. Lett. 67, 614-619 (2004).

[2] S. Pochon, K. F. MacDonald and N. I. Zheludev, "Controlling the optical properties of gallium nanoparticles with a beam of electrons." Phys. Rev. Lett. 92, 145702 (2004). 